\title{
Resiliencia y Envejecimiento
}

\author{
Juan de D. Uriarte Arciniega \\ Universidad del País Vasco (España)
}

La resiliencia fue un concepto introducido en el estudio de las respuestas y la adaptación positiva de personas que vivían en entornos de riesgo, en contextos y situaciones que suponían amenazas potenciales al desarrollo normal como la pobreza, la enfermedad y la violencia. Posteriormente se ha introducido en el estudio de los fenómenos del desarrollo y los procesos que ocurren en todas las etapas y edades del ciclo vital, incluidas las etapas más tardías. La perspectiva de la resiliencia ha modificado el anterior modelo del envejecimiento que lo asociaba con pérdidas por otro más integral en el que las personas mantienen altos niveles de funcionamiento positivo, a pesar de las circunstancias adversas, las limitaciones y los deterioros. Esta concepción es convergente con el enfoque de la resiliencia y con la idea de que el resiliente es más una persona normal que alguien excepcional. Dos procesos de tipo psicobiológico dan sustrato a la resiliencia psicológica en la vejez: la neuroplasticidad y la psiconeuroinmunología. Para finalizar, se destacan los recursos personales y requisitos llamados resilientes que contribuyen al envejecimiento satisfactorio, incluyendo la longevidad.

Palabras clave: Resiliencia, envejecimiento, longevidad, neuroplasticidad, psiconeuroinmunología.

Resilience and ageing. Resilience was a concept introduced in the study of responses and positive adaptation of people living in risky environments, contexts and situations that posed potential threats to normal development like poverty, disease and violence. Subsequently introduced in the study of developmental phenomena and processes that occur in all ages and stages of the life cycle, including the later stages. The resilience's perspective has modified the previous model of aging that associated losses with a more comprehensive in that people have high levels of positive functioning, despite adverse circumstances, limitations and impairments. This conception is convergent with the approach of resilience and the idea that a resilient person is normal, isn't exceptional. Two psychobiological processes support psychological resilience in old age: neuroplasticity and psychoneuroimmunology. To finish, are highlighted the personal resources and requirements called resilient contributing to successful aging, including longevity.

Keywords: Resilience, ageing, longevity, neuroplasticity, psychoneuroimmunology.

Correspondencia: Juan D. Uriarte Arciniega. Universidad del País Vasco /EHU. B ${ }^{\circ}$ Sarriena, s/n. C.P.: 48994. Leioa-Bizkaia (España). E-mail: juandedios.uriarte@ehu.es 


\section{La resiliencia en la vejez}

En todas las etapas del ciclo vital las personas están necesitadas de realizar ajustes y regulaciones en sus pensamientos, conductas, creencias y relaciones, con el objetivo de lograr una mejor y más satisfactoria adaptación consigo mismo y con su entorno. Actualmente se admite que el desarrollo individual no se produce con ausencia de dificultades sino a pesar de las dificultades. En ocasiones, las dificultades pueden ser muy grandes y suponer barreras al desarrollo normal, como en el caso de algunas discapacidades. En otras, son oportunidades para poner en juego nuevos recursos y soluciones. La dinámica de las ganancias y las pérdidas ante las situaciones complicadas de la vida es parte del desarrollo normal.

Las diversas definiciones de la resiliencia la entienden como la habilidad para adaptarse positivamente a las situaciones de adversidad (Uriarte, 2005). El paradigma de la resiliencia se introdujo en la etapa de la vejez a partir de la década de los 90 (Staudinger, Margiske y Baltes, 1995). Esta perspectiva modificó el anterior modelo que asociaba envejecimiento con pérdidas, destacando que las personas mayores mantienen y manifiestan altos niveles de funcionamiento adecuado a pesar de las circunstancias adversas y de las pérdidas.

Los procesos reguladores y de adaptación identificados con la resiliencia están presentes en todas las edades de la vida, aunque no sean los mismos en todas las edades. En la vejez la resiliencia resulta del funcionamiento "ordinario", normativo, no excepcional, de recursos y procesos de autorregulación: relaciones significativas y apoyos sociales, oportunidades para el éxito, actitudes positivas, conviviendo en entornos saludables. En consecuencia, los problemas aparecen y tienden a persistir cuando esos sistemas de regulación y adaptación humanas no son prioritarios o están alterados como consecuencia de la adversidad.

El envejecimiento es una etapa del ciclo vital que en las sociedades desarrolladas está adquiriendo un gran protagonismo, en parte debido a la ampliación de la esperanza de vida. Cada vez más las personas viven su vejez con plenitud y personas longevas se desenvuelven con notable calidad de vida subjetiva. La genética no es la única ni la parte más responsable del envejecimiento, aunque se reconoce que el envejecimiento tiene un componente de género: las mujeres tienden a ser más longevas que los varones, por término medio.

Es indudable que la vejez comporta cambios físicos, sociales y psicológicos notables. También se producen adversidades que son generadoras de estrés: la muerte de seres queridos, las limitaciones físicas y sensoriales, el decline del estatus profesional y otras disminuciones, que pueden ser consideradas como normativas para las personas ancianas, entendiendo por normativas las que afectan a todas las personas del mismo rango de edad o etapa. Envejecer es un proceso natural e irreversible, no es un proceso patológico, ni una enfermedad, ni una mera involución, aspectos susceptibles de 
tratamiento médico o psicológico. Cosa distinta es que hay enfermedades originadas en etapas anteriores que sí requieren de más cuidados especializados.

La resiliencia es, por lo tanto, el constructo que denomina al proceso mediante el cual las personas realizan una integración positiva, a pesar de las dificultades y limitaciones que se producen en la vejez, y alcanzan el final de la vida consciente de sí mismas, con bienestar emocional, satisfechas con su vida. Así la resiliencia es una faceta del propio envejecimiento.

Por resiliencia entendemos el mantenimiento de la identidad personal a pesar de los cambios biológicos, sociales, psicológicos, y a pesar de los deterioros; es resiliencia la manera de compensar y modificar las habilidades físicas, sensoriales y cognitivas que manifiesten algún declive, así como el modo de afrontar las estigmas sociales que pueden existir sobre el envejecimiento: sujeto no productivo, estorbo, mantenido, enfermo, loco, inservible, irrecuperable, pendiente de que se muera; es un envejecimiento resiliente el modo de vivir el presente y el futuro con optimismo, sin añoranzas por el pasado, para seguir sintiendo, pensando, queriendo, participando y autorrealizándose.

La perspectiva de la resiliencia coincide con la teoría psicosocial del desarrollo de Erikson (2000) cuando advierte de los procesos psicológicos positivos que hacen las personas mayores: la autoaceptación de sí mismo, la aceptación de los cambios físicos, sexuales, sociales y laborales; la integración de las etapas pasadas, la renuncia a ciertos objetivos de etapas pasadas, aspectos que contribuye a disminuir las reacciones emocionales negativa como el estrés, la frustración o depresión, contribuyendo a mantener adecuados niveles de salud, de bienestar, de participación social y comunitaria, que aportan calidad de vida subjetiva.

\section{Plasticidad, sistema inmunológico y resiliencia en la vejez.}

La resiliencia se ha identificado con una característica básica del desarrollo humano como es la plasticidad. La plasticidad neuronal es la característica que explica la adaptación funcional del sistema nervioso para minimizar los efectos de las alteraciones estructurales o fisiológicas, cualquiera que sea la causa originaria. En cualquier momento de la vida, el cerebro tiene capacidad de cambio estructuralfuncional por influencias endógenas y exógenas. La plasticidad cerebral en la vejez es menos notoria que en etapas anteriores, pero se ha puesto de manifiesto que a la par que ocurren ciertos declives pueden ocurrir incrementos en otros dominios (Pascual-Castroviejo, 1996; Triadó, 2001).

La neuroplasticidad es la denominación del fenómeno investigado recientemente en el campo de las neurociencias que viene a demostrar que la actividad mental produce un aumento de las neuronas y modificaciones en el cerebro según el tipo de actividad de la que se trate, dando así fundamento científico a los programas de 
mantenimiento y mejora de la memoria, la inteligencia y otros aprendizajes complejos en las edades avanzadas (Navarro y Calero, 2006).

Si la plasticidad es la capacidad de desarrollar los procesos cerebrales necesarios que permiten una continuada adaptación al medio, la resiliencia en la vejez no sería más que la expresión positiva y psicológica de esta característica adaptada a la circunstancias personales y sociales. Se sabe que la participación activa del sujeto es fundamental en la dirección e intensidad del cambio cerebral, en la eficacia y la satisfacción. La plasticidad indica, también, que el sujeto posee capacidades de reserva, recursos a los que se puede acceder en determinados casos como en los procesos degenerativos masivos (Staudinger y Fleesson, 1996).

Para estimar la esperanza de vida de las personas, actualmente cuenta más la edad biológica que la cronológica. La edad biológica está determinada por el sistema inmunológico, que es el mejor marcador de la esperanza de vida y de la longevidad (Alonso y de la Fuente, 2008). Así, cuanto menor es la edad biológica y los marcadores inmunológicos son más parecidos a los de una persona joven, mayor es la probabilidad de vivir más años.

Es importar recordar que el sistema inmunológico depende menos de la genética que de las condiciones y estilo de vida del sujeto, y que no evoluciona solo sino que depende del sistema nervioso y de las emociones y pensamientos con el que está íntimamente relacionado. Hace tiempo que Adler (1974) demostró la posibilidad de influir y condicionar el sistema inmunológico y el sistema nervioso mediante intervenciones netamente psicológicas.

Está demostrado que nuestros estados emocionales positivos y negativos inciden respectivamente en la disposición a contraer enfermedades y en la manera de recuperarnos de ellas. Se sabe, por ejemplo, que el aislamiento social, tan frecuente en personas de avanzada edad, produce un declive funcional, cognitivo e inmunológico que compromete la salud y la esperanza de vida (Arranz, Giménez-Llort, De Castro, Baeza, De la fuente, 2009). Al mismo tiempo, las experiencias de superación del estrés emocional, el enriquecimiento cognitivo y los afectos positivos, fortalecen el sistema inmunitario y aumentan la longevidad (De la Fuente. 2009). En definitiva, un sistema inmunológico bien regulado, en el que las cualidades psicológicas positivas representadas por la resiliencia sean predominantes, contribuirá a retrasar el envejecimiento biológico y a favorecer la longevidad.

\section{El envejecimiento satisfactorio y la resiliencia}

Rowe y Kakn, (1997, citado en Lamand, Depp, Alliso, Laner et al., 2008) identificaron 7 componentes del llamado envejecimiento satisfactorio: ausencia de discapacidad; funcionamiento cognitivo alto/bueno; inserción social; bienestar percibido; envejecimiento satisfactorio percibido; actitud positiva; autonomía y control. Las 
investigaciones posteriores confirman las condiciones que contribuyen al envejecimiento satisfactorio, incluyendo la longevidad:

1.- Estilo de vida activo continuado. Se refiere a la práctica de las actividades comunes de la vida diaria para el autocuidado personal, el cuido del entorno y las actividades asociadas. Mantenerse activo no es solo moverse físicamente y hacer ejercicio deportivo. También es trabajar y mantener actividades culturales y recreativas. La actividad, entendida como rasgo de personalidad, correlaciona positivamente con la longevidad (Terracciano, Corinna Löckenhoff, et al., 2008). La resiliencia se relaciona con la actividad físico y la promoción de la salud. El descenso habitual de la actividad física se puede compensar con un aumento de la actividad mental y social. La dieta saludable, el autocuidado, el ejercicio, la ausencia de molestias físicas, son claves para el bienestar y la satisfacción en la vejez.

2.- Participación social: Las personas resilientes están más interesadas en la participación social (Butler y Ciarrochi, 2007), en mantener las redes familiares, sociales y las amistades. Estar con personas, relacionarse positiva y significativamente con ellas, y particularmente si son más jóvenes, es un factor de resiliencia. La participación social, los afectos y la autoestima positiva, son procesos que se retroalimentan. Diversificar los apoyos y las relaciones es además una fuente de estimulación sociocognitiva. Tener una personalidad extravertida y mantener relaciones permiten la comunicación, el desarrollo y mantenimiento de las habilidades sociales y comunicativas: expresar ideas y sentimientos, escuchar a los demás, dialogar, mantener la atención, resolver conflictos, etc.

No hay suficientes evidencias y puede ser un tópico que en la $3^{\mathrm{a}}$ y $4^{\mathrm{a}}$ edad se produce una reducción de los contactos sociales, aislamiento y soledad. Los datos empíricos muestran una relativa estabilidad de los contactos sociales a lo largo de la vida, si se excluyen los contactos laborales y profesionales. Las personas más mayores, por lo generan, no se quejan de la soledad o no manifiestan que en la vejez estén más solas que cuando eran más jóvenes, excepto en aquellos casos en los que los contactos sociales se limitaban a la pareja y esta ha fallecido. En este supuesto, la viudez sería un estado de riesgo de desajuste social, que unas veces lleva al aislamiento y otras a un incremento de la actividad social, tras superar la dependencia del cónyuge.

3.- Recreación educativa. El mayor tiempo libre de responsabilidades y de trabajos no deseados facilita la recreación educativa en la que las personas no tratan de "pasar el rato", "matar el tiempo", "dar vueltas" sino de vivir en el tiempo y disfrutar con lo que se hace. Para ello es importante implicarse en procesos de aprendizaje, de participación activa en la cultura, no solo en actividades contemplativas o de espectador distante en excursiones o exposiciones. La participación mental, la disposición para seguir aprendiendo, el interés por las personas y las situaciones del entorno, en mayor medida que las actividades de autorreflexión o de recuerdo de su vida pasada, son 
aspectos relacionados con la resiliencia porque mantienen y potencian las capacidades. Lo que lleva antes a la "muerte" es el desinterés por lo que rodea y la falta de actitud biófila.

4.- Aceptar las transformaciones. Las pérdidas que ocurren en el envejecimiento son un desafío que exige acciones para compensar pérdidas con ganancias. La aceptación de las transformaciones permite el mantenimiento de la identidad y el autoconcepto general. Se sabe que un elemento del autoconcepto positivo es la autoaceptación corporal. La actitud positiva hacia la propia edad influye en la longevidad saludable (Levy, Slade, Kunkel, y Kasl, 2002; Vailand y Mukamal, 2001). La aceptación psicológica de los cambios corporales contribuye a disminuir la reacción emocional negativa que puede derivar de la falta de agilidad y fuerza, la lentitud y baja productividad, la hipoacusia, las arrugas visibles. Envejecer bien significa aprender a valorar aspectos personales nuevos o poco valorados hasta entonces, a tener en cuenta las limitaciones sin amarguras, a aceptar la disminución del rendimiento en algunas áreas como lo físico y corporal, cuando se puede ganar en lo subjetivo y en lo emocional, en sabiduría y profundidad de los sentimientos. Aceptar las transformaciones es construir una autoestima con el yo presente, incluido el yo corporal

5.- Tener proyectos de vida en la vejez. Decimos que los proyectos son los ideales del yo actualizado, los que dan sentido al día a día. Terminar un día con la idea de que al día siguiente uno tiene cosas importantes en la que debe implicarse. Unos son proyectos inmediatos como cuidar la casa, ver a los nietos, desplazarse para un proyecto diario que organiza la vida alrededor de ciertas rutinas. Otros son más amplios y no se centran en el yo sino en los suyos: ver los proyectos e ilusiones que logran sus seres queridos.

6.- Recordar con proyección al presente y al futuro, no como mera añoranza del pasado. Estar en paz con el pasado, olvidar conscientemente, perdonar y perdonarnos: los remordimientos, el rencor o la culpabilidad, son venenos que impiden a las personas avanzar (Knopoff, Santagostino y Zarebski, 2004).

7.- Gestionar las emociones y sentimientos para poder cuestionar los prejuicios, para reírse de uno mismo, para relativizar los asuntos sin caer en pensamientos deterministas y absolutistas, de "todo y nada". Evitar aferrarse a una pretendida identidad única: "yo soy así", "siempre he sido así", que pone barreras a la adaptación y a los cambios. La estabilidad emocional y el bajo nivel de neuroticismo es una característica de la personalidad que está asociada a vivir más años (Terracciano et al., 2008).

8.- No vivir toda la vida sostenido por el otro, "soy la mujer de... el padre de...," o en una función o parte del yo: "yo soy mecánico", o en un rol de..., como bastón de identidad y que implicaría la quiebra ante la eventualidad de su pérdida. 
9.- Desarrollar la generatividad, ser productivo y creativo en función de la siguiente generación. Y el sentido de la trascendencia, puesto que la vida se inserta en procesos más amplios del espacio y tiempo actuales, que para muchas personas da sentido a la vida presente, como si cada uno fuera parte de un proyecto más amplio, que le trasciende. Es como dar sentido al proyecto humano, por lo menos, aunque algunos entienden la trascendencia desde una perspectiva religiosa.

10.- Aceptar el fin de la vida, la muerte, vivir hasta la muerte, no hacia la muerte. Reflexionar sobre la muerte es una exigencia de todas las personas. Posiblemente hace que la vida tenga un mayor sentido.

\section{Los recursos personales que potencian la resiliencia durante el} envejecimiento

Uno de los rasgos de personalidad que en mayor medida contribuyen al envejecimiento satisfactorio y la longevidad es la Conciencia o Responsabilidad (Chapman, Roberts y Duberstein, 2011; Terracciano et al., 2008). Las personas que poseen esta característica en grado alto son cuidadosas, ordenadas, responsables de sí mismas y de llevar a cabo conductas saludables, comprometidas por hacer las cosas bien, evitando las situaciones y conductas de riesgo para su salud y su bienestar. Un exceso de preocupación por la salud, que puede llevar a desarrollar conductas neuróticas, obsesivas o de otra índole y que generan preocupaciones innecesarias, es claramente contraproducente.

Se han identificado los recursos personales que se activan ante ciertas situaciones estresantes o de riesgo, permitiendo resultados positivos y el bienestar en todas las edades, no solamente durante el envejecimiento.

1. La identidad positiva y sentido de autoeficacia

La autoeficacia es sinónimo de la percepción de sí mismo positiva y la confianza en sus cualidades y posibilidades. Bandura (1999) señaló que la expectativa de autoeficacia no dice nada con respecto al número de capacidades que uno posee, pero sí de lo que garantiza que puede hacer en circunstancias diversas.

Es la creencia de que con las propias capacidades uno puede organizar y modificar correctamente las situaciones presentes y futuras. Se relaciona con el locus de control interno. Ayuda a generar seguridad en sí mismo, bienestar, optimismo; activa y optimiza recursos, particularmente para hacer frente a las amenazas y al estrés. Del mismo modo, facilita y mantiene los contactos sociales y el apoyo social. La resiliencia correlaciona negativamente con la percepción de limitaciones y problemas en el funcionamiento mental propio que el sujeto no los compensa (Lamond et al., 2008).

2. El control personal

Ser uno mismo el que gestiona el curso de su vida es una característica compartida por personas resilientes y personas longevas saludables. Esta característica 
en más determinante incluso que la dieta, el ejercicio, la predisposición genética o la ausencia de estrés (Mouratidis y Price, 2010). El sentido de control sobre la vida propia y sobre los acontecimientos en los que participamos, sea efectivamente real o percibido como tal, juega un papel muy importante en los procesos de cambio y en la adaptación externa a los cambios y a las crisis. El sentido de control interno influye en el establecimiento de las metas personales, optimiza las motivaciones y los recursos emocionales, mantiene el control sobre el ambiente y realza lo necesario para alcanzar las metas propuestas (Heckausen, 2001).

3. Las emociones positivas

Las personas mayores suelen generan más actitudes y emociones positivas que negativas. Son más tolerantes con los demás, menos prejuiciosas, menos rencorosas o vengativas. Animan a buscar acuerdos, evitan los conflictos si son conscientes de ellos. La gratitud, el amor, el perdón, la solidaridad, el altruismo, la alegría, entre otras, son emociones positivas que procuran experiencias agradables y de bienestar, sentimientos de paz interior, de realización personal. Las emociones positivas tienen un alto valor de resiliencia propia y ayuda a generar resiliencia en el entorno.

4. La actitud positiva y el optimismo vital

El optimismo es una fortaleza psicológica y un factor protector ante las dificultades. Es la actitud general de que la vida merece ser vivida plenamente y la creencia de que en el futuro ocurrirán resultados favorables. Es una actitud que sirve para enfocar los acontecimientos vitales desde la perspectiva que acentúa los aspectos favorables y minimiza los negativos, tanto si se refieren a sí mismos como a las personas queridas de su entorno. Ante las dificultades, las expectativas favorables de las personas optimistas incrementan los esfuerzos para alcanzar los objetivos, mientras que las expectativas desfavorables reducen tales esfuerzos o llevan a abandonar la tarea (Riolli, Savicki y Cepani, 2006). Estas actitudes parece que tienen resultados beneficiosos para la salud física y mental. En el envejecimiento satisfactorio el optimismo es elevado (Rius, 2012).

\section{Estilo de afrontamiento}

Si el afrontamiento se refiere al modo en que el sujeto maneja los retos o amenazas, para algunos la resiliencia es ya un estilo de afrontamiento. Las personas mayores recurren más a un afrontamiento centrado en las emociones que centrado en el problema. Las experiencias de afrontamiento acumuladas le sirven para adaptarse a los diferentes problemas y a las situaciones estresantes. El afrontamiento es el medio de acomodación del sujeto que evita y reduce las discrepancias entre el individuo, las metas, las creencias y las relaciones con los demás.

\section{La espiritualidad}

Ha sido comprobado que la espiritualidad es un factor muy importante en el afrontamiento y la adaptación a los cambios y las dificultades, en el sentido del bienestar 
y, en definitiva, en el proceso de la resiliencia (Rivera y Montero, 2005; San Martín, 2008). Aunque hay una evolución de la espiritualidad a lo largo de la vida, para los ancianos actualmente sigue siendo un factor de resiliencia importante, principalmente en su vertiente religiosa.

\section{CONCLUSIONES}

Cada etapa del desarrollo supone riesgos, amenazas, dificultades, oportunidades, recursos, situaciones que, en definitiva, requieren de procesos resilientes. Lo mismo se ha de producir en la etapa de la vejez. No hay evidencia de que ser persona resiliente en etapas anteriores (niñez, adolescencia, adultez) garantice serlo en la vejez. Del mismo modo, no hay que confundir envejecimiento normal y anciano resiliente con persona longeva. La longevidad no es exactamente lo mismo que la resiliencia. Hay una longevidad "patológica", cuando la persona está afectada por trastornos neurológicos (demencias, Alzheimer,) y psicológicos (depresión) que dificultan o impiden una vida con sentido o significado.

Aquellos mayores que han sabido ajustarse personal y socialmente a los cambios y a las adversidades, normales y extraordinarias, que siguen teniendo un adecuado nivel de funcionamiento en las diferentes áreas vitales del desarrollo, que mantienen una adecuada satisfacción con su vida, bienestar psicológico, son lo que podríamos denominar "mayores resilientes". Su estudio aporta orientaciones para la intervención y la prevención con personas menos resilientes.

Según Suarez Ojeda y Melillo (2003), la resiliencia en el envejecimiento tiene mucha relación con la calidad del narcisismo, cuando los aspectos estructurantes de la identidad cambian (la imagen corporal, la sexualidad, los roles sociales, las funciones, etc.) y a pesar de ello la persona conserva la identidad propia. En ese caso, la resiliencia y el envejecimiento normal (no patológico) son coincidentes.

Tras llevar a cabo una investigación con muestras muy amplias, Zengl y Shen (2010) concluyeron que los centenarios son mucho más resistentes que cualquier otro grupo de edad de la población de mayores y que la resiliencia, media por cuestionarios, es la característica que contribuye significativamente a la longevidad. Estos resultados no sólo tienen importancia científica sino también social y política, pues dan soporte a los programas destinados a promocionar la resiliencia entre los mayores.

\section{REFERENCIAS}

Alonso, A. y De la Fuente, M. (2008). Marcadores inmunológicos de envejecimiento. Revista Española de Geriatría y Gerontología, 43(3), 167-179. 
Arranz, L., Giménez-Llort, L., De Castro, N.M., Baeza, I. y De la Fuente, M. (2009). El aislamiento social durante la vejez empeora el deterioro cognitivo, conductual e inmunitario. Revista Española de Geriatría y Gerontología, 44(3), 137-142.

Bandura, A. (1999). Ejercicio de la eficacia personal y colectiva en sociedades. En A. Bandura, (coord.), Auto eficacia: Cómo afrontamos los cambios en la sociedad actual (pp.19-54). Bilbao: Desclée de Brouwer.

Butler, J. y Joseph Ciarrochi, J. (2007). Psychological acceptance and quality of life in the elderly. Quality of Life Research, 16, 607-615.

Chapman, B.P., Roberts, B. y Duberstein, P. (2011). Personality and longevity: knowns, unknowns, and implications for public health and personalized medicine. Journal of Aging Research, 10, 759170.

De Fuente, M. (2004). Envejecimiento desde la biología. ¿Conocemos los límites? Humanitas. Monografía 1, 39-51.

De la Fuente, M. (2009). Teorías del envejecimiento. En G. Varela y E. Alonso (coord.): Retos de la nutrición en el siglo XXI ante el envejecimiento poblacional. Cap. 2, (pp. 29-48). Instituto Tomás Pascual Sanz para la nutrición y la salud. Universidad San Pablo Ceu. Madrid.

Erikson, E. (2000). El ciclo vital completado (edición revisada). Barcelona: Paidós.

Heckhausen, J. (2001). Adaptation and Resilience in Midlife. En M.E. Lachman, (Ed), Handbook of midlife development. Wiley series on adulthood and aging (pp. 345-391). Hoboken, NJ, US: John Wiley \& Sons Inc.

Knopoff, R., Santagostino, L. y Zarebski, G. (2004). Resiliencia y envejecimiento. En A. Melillo, E.N. Suarez Ojeda y D. Rodríguez (Comp.), Resiliencia y subjetividad (pp. 213- 228). Buenos Aires: Paidós.

Lamond, A., Depp, C., Allison, M., Langer, R., Reichstadt, J., Moore, D., Golshan, S., Ganiats, T. y Jeste, D. (2008). Measurement and predictors of resilience among communitydwelling older women. Journal of Psychiatric Research, 43(2), 148-154.

Levy, B.R., Slade, M.D., Kunkel, S.R. y Kasl, S.V. (2002). Longevity increased by positive selfperceptions of aging. Journal of Personality and Social Psychology, $\mathrm{n}^{\circ}$ 83(2):261-270.

Luthar, S.S., Cicchetti, D. y Becker, B. (2000). The construct of resilience: A critical evaluation and guidelines for future work. Child Development. 71(3): 543-562.

Mouratidis, H. y Price, G. (2010). They Live Longer. The Secrets of Healthy and Active NinetyYear-Olds. Recuperado de: www.TheyLiveLonger.com.

Navarro, E. y Calero, M.D. (2006). La plasticidad cognitiva en la vejez. Técnicas de evaluación e intervención. Barcelona: Octaedro.

Pascual-Castroviejo, I. (1996). Plasticidad cerebral. Revista de Neurología, 24(135), 1361-1366.

Riolli, L., Savicki, V. y Cepani, A. (2006). Resilience in the face of catastrophe: optimism, personality, and coping in the Kosovo crisis. Journal of Applied Social Psychology, 32(8), 1604-1627.

Rius, N. (2012). Psychosocial, behavioral, and biological aspects of optimism. Psychosocial and biological aspects of dispositional optimism at old age (cap. 8). Tesis Doctoral. Leiden University. Recuperado de: https://openaccess.leidenuniv.nl/bitstream/handle/1887/19762/Back.pdf?sequence=13

Rivera, A. y Montero, M. (2005). Espiritualidad y religiosidad en adultos mayores. Salud Mental, 28(6), 51-58.

Rowe, J.W. y Kahn, R.L. (1997). Successful aging. Gerontologist, 37(4), 433-440.

San Martín, C. (2008). La espiritualidad en el proceso de envejecimiento del adulto mayor. Hologramática, 8(1),103-120. 
Staudinger, U.M. y Fleeson, W. (1996). Self and personality in old and very old age: A sample case of resilience? Development \& Psychopathology, 8, 867-885.

Staudinger, U.M., Marsiske, M. y Baltes, P.B. (1995). Resilience and reserve capacity in later adulthood: Potentials and limits of development across the life span. En D. Cicchetti y D. Cohen (Eds.), Manual of Developmental Psychopathology, 2, 801-847. New York: Wiley. Recuperado de: http://www.midus.wisc.edu/findings/pdfs/95.pdf

Terracciano, A., Löckenhoff, C.E., Zonderman, A.B., Ferrucci, L. y Costa, P.T. Jr. (2008). Personality predictors of longevity: Activity, emotional stability, and conscientiousness. Psychosomatic Medicine, 70(6), 621-627.

Triadó, C. (2001). Cambio evolutivo, contextos e intervención psicoeducativa en la vejez. Contextos Educativos, 4, 119-133.

Uriarte, J.D. (2005). La resiliencia. Una nueva perspectiva en psicopatología del desarrollo. Revista de Psicodidáctica, 10(2), 61-80.

Uriarte, J.D. (2013). La perspectiva comunitaria de la resiliencia. Revista de Psicología Política, 47, 7-18.

Vaillant, G.E. y Mukamal, K. (2001) Successful Aging. American Journal of Psychiatry, 158(6), 839-847.

Zeng, Y. y Ke Shen, K. (2010). Resilience Significantly Contributes to Exceptional Longevity. Current Gerontology and Geriatrics Research; 2010: 525693. Published online 2010 December 6. http://www.hindawi.com/journals/cggr/2010/525693/.

Recibido: 24 de marzo de 2014

Recepción Modificaciones: 26 de mayo de 2014

Aceptado: 30 de mayo de 2014 